\title{
A Survey Paper on E-Learning Recommender System
}

\author{
Reema Sikka \\ M.Tech (SE)-II \\ University Institute of \\ Engineering and Technology \\ MDUHaryana India
}

\author{
Amita Dhankhar \\ Associate Professor \\ University Institute of \\ Engineering and Technology \\ MDU Haryana India
}

\author{
Chaavi Rana \\ Associate Professor \\ University Institute of \\ Engineering and Technology \\ MDU Haryana India
}

\begin{abstract}
A recommender system in an e-learning context is a software agent that tries to "intelligently" recommend actions to a learner based on the actions of previous learners. This recommendation could be an on-line activity such as doing an exercise, reading posted messages on a conferencing system, or running an on-line simulation, or could be simply a web resource. These recommendation systems have been tried in ecommerce to entice purchasing of goods, but haven't been tried in e-learning. This paper suggests the use of web mining techniques to build such an agent that could recommend online learning activities or shortcuts in a course web site based on learners' access history to improve course material navigation as well as assist the online learning process. These techniques are considered integrated web mining as opposed to off-line web mining used by expert users to discover online access patterns
\end{abstract}

\section{Keywords}

E-learning, Recommender Systems, Collaborative Filtering, Content-based filtering.

\section{INTRODUCTION}

E-learning is indeed a revolutionary way to provide education in life long term, comparing with the traditional face-to-face style teaching and learning. Nowadays more and more people have benefited from various e-learning programs. However, high diversity of the learners on the Internet poses new challenges to the traditional "one-size-fit-all" learning model, in which a single set of learning resource is provided to all learners. In fact, the learners could have various interests; even sharing with the common interests, they may have different levels of expertise, and hence they cannot be treated in a uniform way. It is of great importance to provide a personalized system which can automatically adapt to the interests and levels of learners.

Recommender Systems are implemented in commercial and non-profit websites to predict the user preferences. The main functions of recommendation systems include analyzing user data and extracting useful information for further predictions A recommender system is a piece of software that helps users to identify the most interesting and relevant learning items from a large number of items. Recommender systems may be based on collaborative filtering (by user ratings), contentbased filtering (by keywords), and hybrid filtering (by both collaborative and content-based filtering). The following figure is a general model of recommendation which summarizes the recommendation process by identifying the communication among its components. A recommendation seeker can be considered as a user of the system. The seeker may directly ask for a recommendation, or the recommender may produce recommendations without the seeker's demand. Seekers may get into contact with preference provider and represent some information about their preferences. If not, the recommender requests preference information from the user explicitly. Based on the known preferences of the seeker and those of other people, the recommender recommends items the seeker probably will like. The seeker may use the recommendation to select items from the universe of alternatives or to communicate with like-minded other users as the recommender also identifies people with similar interests.

\subsection{Recommendation Systemsfor E- Learning}

An "e-learning task recommender" is a recommendation system that would recommend a learning task to a learner based on the tasks already done by the learner and their successes, and based on tasks The similarity of the learners could be established using user profiles, or could be based on common previous access patterns made by other "similar" learners. The similarity of the learners could be established using user profiles, or could be based on common previous access patterns. In principle, there are two major parts in the design of such an agent: a "learning" module that learns from past access patterns and infers an individual or common access model; and an "advising" module that applies the learned model at given times to recommend actions. There are many ways to implement this process, such as data clustering, association rule mining, or collaborative filtering [11] etc.

Software agents that recommend actions, products, or other items have been used in some applications. However, to the best of our knowledge there is no distance learning system to date that provides such automated facilities to automatically suggest learning activities or resources. In the field of electronic commerce, however, given the lucrative prospects, a significant research effort has been made to devise elaborate methods to take advantage of customers' accesses and purchase behaviours in order to enhance the purchasing experience and customer satisfaction by user profiling and smart recommendations, and thus increase profit. Recommenders are used to boost sales by displaying products or services a consumer is likely to be interested in. Systems for recommendation such as Amazon.com that suggests books or other products to purchase related to a current purchase based on preference information and other users purchases. The techniques used are, however, very simple and not always accurate or even effective. Basically, the program compares the set of items purchased by the current customer with the set of items purchased by other customers, selects the customers with the bigger item overlap with the current customer's item set, then finally picks some items not yet bought by the customer but present in the baskets of customers with high overlap and presents them as a recommendation list to the current customer. More sophisticated methods take into account ratings of products given by customers and select 
products for recommendation from customers that rate items the same way or level as the current customer. This technique also used in information retrieval for retrieving text documents that are similar is called collaborative filtering [11]. Recommendation of movies or music compact discs, such as moviefinder.com, uses collaborative filtering by predicting a person's preferences as a linear weighted combination of other people's preferences. Since we do not have ratings for course material, collaborative filtering is not applicable for devising an accurate recommender agent for elearning activities. Moreover, we are interested in recommending beneficial learning activities to enhance online learning, as well as recommending shortcuts or jumps to some resources to help users better navigate the course materials.

\subsubsection{What Makes Recommendations Different in} E-learning from Other Domains

An e-learning recommender system includes:

- Items liked by learners might not be pedagogically appropriate for them. For example, a learner without prior background on the techniques of web mining may only be interested in knowing the state-of-theart of web mining techniques in ecommerce. Then, it should be recommended that he/she read some review papers. For example, an editorial article by two of the leading researchers in this area [5], although there are many high quality technical papers related to his/her interest. On the other hand, for the learner coming from industry with some prior knowledge who wants to know how web mining can be utilized to solve e-commerce problems, should be recommended, because the paper is the KDD-Cup 20001 organizers' report on how web mining can support business decision making for a real-life e-commerce vendor, and points out challenges, as well as lessons learned from the competition, which can benefit both researchers and industry practitioners. By contrast in other domains, recommendations are made based purely on users' interests.

- Customization should not only be made about the choice of learning items, but alsoabout their delivery.For example, some instructors will recommend learners to read an interesting magazine article, such as a related article in Communications of ACM, before a technical paper, because they believe it will help learners understand the technical paper and make them less intimidated. However, this is not the case in ecommerce recommendations, where site managers prefer to leave the list of recommended items unordered to avoid leaving an impression that a specific recommendation is the best choice.

\subsubsection{Recommender System Approaches}

Recommender system can be built with many approaches. Below are some of them:

- Random prediction algorithm is an algorithm that randomly chooses items from the set of available items and recommends them to the user. Since the item's selection is done randomly, the accuracy of the algorithm is based on luck; the greater the number of items is the chance of good selection lowers. Random prediction has a great probability of failure. Thus, it has never been taken seriously by any researcher or vendor and only serves as reference point 1 , helping to compare the quality of the results obtained by the utilization of a more sophisticated algorithm [15].

- Frequent sequences can help build recommender systems. For example, if a customer frequently rates items we can use the frequent pattern to recommend other items to him. The only problem is that this method will only be efficient after the customer makes minimum purchases.

- Collaborative filtering algorithms (CF) are algorithms that require the recommendation seekers to express their preferences by rating items. In this algorithm, the roles of recommendation seeker (a user) and preference provider are merged; the more users rate items (or categories), the more accurate the recommendation becomes. In most $\mathrm{CF}$ approaches, there is a list of users $U=u 1, u 2,,, u_{m}$ and a list of items $I=i 1, i 2,,,, i_{n}$. Each user ui has a list of item Iui on which he has expressed his opinion [16].

- Content based algorithms are algorithms that attempt to recommend items that are similar to items the user liked in the past. They treat the recommendation's problem as a search for related items. Information about each item is stored and used for the recommendations. Items selected for recommendation are items that content correlates the most with the user's preferences [17]. For example, whenever a user rated an item, the algorithm constructs a search query to and other popular items by the same author, artist, or director, or with similar keywords or subjects [14]. Content based algorithms analyse item descriptions to identify items that are of particular interest to the user.

\section{LITERATURE SURVEY}

Recommender system in an e-learning context is a software agent that tries to "intelligently" recommend actions to a learner based on the actions of previous learners. This recommendation could be an on-line activity such as doing an exercise, reading posted messages on a conferencing system, or running an on-line simulation, or could be simply a web resource. These recommendation systems have been tried in ecommerce to entice purchasing of goods, but haven't been tried in e-learning. This paper suggests the use of web mining techniques to build such an agent that could recommend online learning activities or shortcuts in a course web site based on learners' access history to improve course material navigation as well as assist the online learning process. These techniques are considered integrated web mining as opposed to off-line web mining used by expert users to discover online access patterns. [1]

The focus in eLearning has recently been shifted from the supporting tools towards the learning person. It has turned out that the learning success can significantly be improved if the learning content is specifically adapted to individual learners' preferences, learning progress and needs. Personalization has evolved as a promising concept to take into consideration individual needs. Due to the dynamic nature of eLearning, this paper focuses not only on static but also particularly on dynamic aspects of personalization. [2] 
A personalized e-learning system which can automatically adapt to the interests and levels of learners is the system which is designed based on the IEEE Learning Technology Systems Architecture (IEEE LTSA) to achieve high scalability and reusability. A feedback extractor with fusion capability is proposed to combine multiple feedback measures to infer user preferences. User profile, which stores user preferences and levels of expertise, is collected by user profiler to deliver personalized information using the collaborative filtering algorithm. [3]

The majority of current web-based learning systems are closed learning environments where courses and learning materials are fixed and the only dynamic aspect is the organization of the material that can be adapted to allow a relatively individualized learning environment. In this paper, we propose an evolving web-based learning system which can adapt itself not only to its users, but also to the open Web. More specifically, the novelty with respect to the system lies in its ability to find relevant content on the web, and its ability to personalize and adapt this content based on the system's observation of its learners and the accumulated ratings given by the learners. Hence, although learners do not have direct interaction with the open Web, the system can retrieve relevant information related to them and their situated learning characteristics. [4]

Recommendation process in distributed information systems extracts similarities and differences between recommendations in stores and the recommendations applied to an e-learning environment. It also explains the phenomena of self-organization and cooperative emergence in complex systems coupled with bio-inspired algorithms to improve knowledge discovery and association rules. Finally, the present recommendation is applied to e-learning by proposing recommendation by emergence in Multi-Agent System architecture. [5]

Recommended learning resources are computed based on the current learner's recent navigation history, as well as exploiting similarities and dissimilarities among learners' preferences and educational content. The proposed framework for building automatic recommendations in e-learning platforms is composed of two modules: an off-line module which pre-processes data to build learner and content models, and an online module which uses these models on-the-fly to recognize the students' needs and goals, and predict a recommendation list. Recommended learning objects are obtained by using a range of recommendation strategies based mainly on content based filtering and collaborative filtering approaches, each applied separately or in combination. [6]

An enormous amount of learning materials in e-learning has led to the difficulty on locating suitable learning materials for a particular learning topic, creating the need for content recommendation tools within learning context. In this paper, we aim to address this need by proposing a novel framework for an e-learning recommender system. Our proposed framework works on the idea of recommending learning materials based on the similarity of content items (using Vector Space Model) and good learners' average rating strategy. This paper presents the overall architecture of the proposed system and its potential implementation via a prototype design. [7]

When building a knowledge-based recommender along the eLearning life cycle, the following issues have to be considered: a) the user interface design of the tools required, b) the process to design/generate the recommendations, c) the process to select the appropriate recommendations, and d) the management of the users' interactions. We are defining a user-centred evaluation approach that copes with those issues and drives the recommender building process in three consecutive steps: 1) elicitation of pedagogically sound recommendations validated by users with a collaborative review, 2) acquisition and validation of the user features to select the appropriate recommendations for the current context, and 3) analysis of the recommendations provided and evaluation of their impact on the us. [8]

Recommender systems have been a useful tool to recommend items in many online systems, including e-learning. However, not much research has been done to measure the learning outcomes of the learners when they use e-learning with recommender system. Instead, most of the researchers were focusing on the accuracy of the recommender system in predicting the recommendation rather than the knowledge gain by the learners. This research aims to compare the learning outcomes of the learners when they use several types of e-learning recommender systems. Based on the comparison made, we propose a new e-learning recommender system framework that uses content-based filtering and good learners' ratings to recommend learning materials, and in turn is able to increase the student's performance. The results show that students who used the proposed e-learning recommender system produced a significantly better result in the post-test. The results also show that the proposed e-learning recommender system has the highest percentage of score gain from pre-test to post-test. [9]

\section{CONCLUSION}

A recommender system is a program that sees what a user is doing and tries to recommend courses of action it thinks would be beneficial to the user. This is the idea behind some systems used in electronic commerce sites to recommend products to customers they might wish to purchase based on their previous purchasing history as well as the purchasing history of those who bought similar goods. To date, this hasn't been proposed for on-line learning environments and no known e-learning system uses such a software agent to enhance the on-line learning experience as described in this paper.

We have proposed an approach to build a software agent that uses data mining techniques in order to build a model that represents on-line user behaviours, and uses this model to suggest activities or shortcuts. These suggestions can help learners better navigate the on-line materials by finding relevant resources faster using the recommended shortcuts and assist the learner choose pertinent learning activities that should Improve their performance based on on-line behaviour of successful learners.

\section{REFERENCES}

[1] Zaiane, O. R.,"Building a Recommender Agent for eLearning Systems",University of Alberta, Edmonton, Alberta, Canada, 2002.

[2] Armin, U., and Kandpal, D., and Tochtermann,K., "First Steps towards Personalization Concepts in eLearning" Know-Centre Graz, 2003.

[3] Li, X., and Chang, S. K., "A Personalized E-Learning System Based on User Profile Constructed Using Information Fusion" University of Pittsburgh, USA, 2006 
[4] Tang, Y. T. ; and McCalla, G.," Smart Recommendation for an Evolving E-Learning System" Dept. of Computer Science, University of Saskatchewan, 2007

[5] Gil, A. B., and García-Peñalvo, F., J., "Learner Course Recommendation in E-Learning Based on Swarm Intelligence", Department of Computer Science, Sciences Faculty University of Salamanca, Salamanca, Spain, 2008.

[6] Khribi, M. K., Jemni, M., \& Nasraoui, O. (2009). Automatic Recommendations for E-Learning Personalization Based on Web Usage Mining Techniques \& Information Retrieval. Educational Technology \& Society, 12 (4), 30-42.

[7] Ghauth, K., I., B., and Abdullah, N., A., " Building an E-Learning Recommender System using Vector Space Model and Good Learners Average Rating" Multimedia University, and University of Malaya, 2009 Ninth IEEE International Conference on Advanced Learning Technologies.

[8] Santos, O.C., and Boticario, J.G., " Building a knowledge-based recommender for inclusive eLearning scenarios" Artificial Intelligence Department, Spain, 2009

[9] Ghauth, K., I., and Abdullah, N., A., " Measuring learner's performance in e-learning recommender systems". Multimedia University, 2010

[10] R. Agrawal, T. Imielinski, and A. Swami. Mining association rules between sets of items in large databases. In Proc. 1993 ACM-SIGMOD Int. Conf. Management of Data, pages207-216, Washington, D.C., May 1993.
[11]S. Chee, J. Han, and K. Wang. Rectree: An efficient collaborative filtering method. In 3rd Int. Conf. On Data Warehousing and Knowledge Discovery (DAWAK 2001), LNCS 2114, pages 141-151, Munich, Germany, September 2001. Springer Verlag.

[12]O. R. Za"ýane and J. Luo. Towards evaluating learners' behaviour in a web-based distance learning environment. In Proc. of IEEE International Conference on Advanced Learning Technologies (ICALT01), pages 357-360, Madison, WI, August 2001.

[13] O. R. Za“ýane, M. Xin, and J. Han.Discovering web access patterns and trends by applying OLAP and data mining technology on web logs. In Proc. Advances in Digital Libraries ADL'98, pages 19-29, Santa Barbara, CA, USA, April 1998

[14]Linden, G., Smith, B., and York, J. Amazon.com recommendations: Item-to-item collaborative filtering. IEEE Internet Computing 7 (2003), 76-80.

[15] Papagelis, M., and Plexousakis, D. Qualitative analysis of user-based and item-based prediction algorithms for recommendation agents. Engineering Applications of Artificial Intelligence 18, 7 (2005), 781-789.

[16] Sarwar, B., Karypis, G., Konstan, J., and Reidl,J. Itembased collaborative filtering recommendation algorithms. In WWW '01: Proceedings of the $10^{\text {th }}$ international conference on World Wide Web (NewYork, NY, USA, 2001), ACM, pp. 285-295.

[22] VAN Meteren, R., and van Someren, M. Using contentbased filtering for recommendation. 